\title{
Cardiac Activity Based on Acoustic Signal Properties
}

\author{
K. CzOPEK* \\ AGH University of Science and Technology, Faculty of Electrical Engineering, Automatics \\ Computer Science and Electronics Department of Automatics, al. A. Mickiewicza 30, 30-059 Krakow
}

The influence of sleeping conditions on human health and well-being is now fully understood, but still underestimated. Sleep disorders e.g. snoring, wheezing or sleep apnea are widespread among people of all ages, although prevalent amidst the male population, obese people with a high value of BMI, people who suffered a stroke, have hypertension or other heart diseases. Medical support is needed in order to ensure a good night's sleep for patients suffering from sleep disorders. Sleep monitoring is also very important in case of emergencies. This research was aimed at finding a correlation between sleep disorders represented by acoustic signals and heart activity parameters. The measurement of breathing through simultaneously acquired acoustic and ECG signals is used to quantify the respiratory obstruction during sleep. The information collected by synchronized recording of acoustic effects and the ECG signal partly overlaps, giving an opportunity to improve accuracy of measurement. Results indicate that the aspect of sleep analysis can be extended using this method. The information collected by simultaneous recording of acoustic effects and the ECG signal is provided and the results are described.

PACS: 43.60.-c, 43.60.+d, 87.19.ug

\section{Introduction}

Obstructive sleep apnea is the most common sleep disorder. During the transition from light sleep to deep sleep, the soft palate muscles and tongue relax. If the tissues in the throat such as the tongue relax too much, vibrations appear and create a sound commonly known as snoring [1]. Figure 1 shows tongue position in various cases: (1) normal unobstructed breathing, (2) partial obstruction-snoring sound appears, (3) complete obstruction-obstructive sleep apnea (OSA) develops. The more narrow the airway, the greater the force of airflow, contributing to increasing tissue vibration. In an extreme case the amount of oxygen one can take in during this blockage can actually drop and become dangerously low.

Sleep apnea is very common, but many people are not aware they suffer from it. One of the most common indicators of sleep apnea is snoring. All snorers show signs of upper airway blockage, but not all snorers have sleep apnea. People who suffer from obstructive sleep apnea may wake up in panic, because they perceive not getting enough breath, gasp. This leads to an increase in adrenaline flow and makes going back to sleep harder. In many cases the sleeper does not even wake up completely and does not know if he or she stopped breathing [8]. The first symptoms of sleep apnea are in fact noticed by patient's life partner or someone else with whom the subject is sharing a bed. Table I shows the response of respiratory, cardiovascular and nervous systems during sleep apnea.

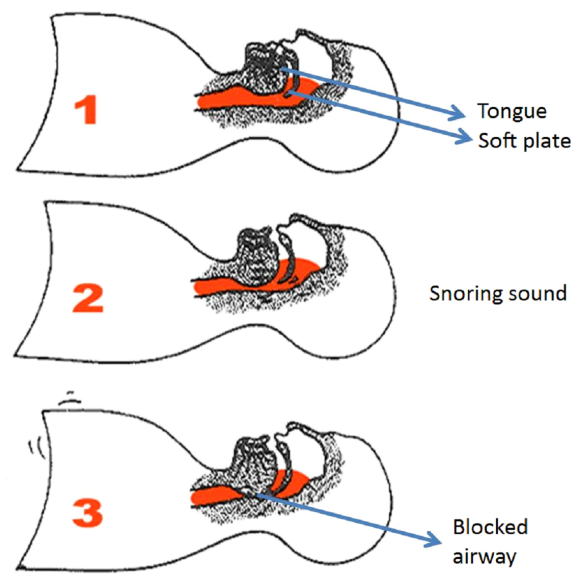

Fig. 1. Tongue positions during sleep.

It is very common that $95 \%$ of people suffering from this ailment are not aware of its existence. People with sleep apnea actually stop breathing while asleep. The lack of breath can last $10 \mathrm{~s}$ or longer and usually happens repeatedly during the night. In each apnea occurrence brain receives signals and interrupts sleep gasping for air. Possible reasons for OSA: obesity, neck circumference, narrowed airway e.g. large tonsils and adenoids in children, high blood pressure, nasal obstruction, drugs, alcohol, nicotine, being male and older, prolonged sitting. Table II shows possible obstructive sleep apnea symptoms.

\footnotetext{
* e-mail: klaudiac@agh.edu.pl
} 
TABLE I

Comparing the response of respiratory, vascular and nervous systems during sleep apnea or immediately after.

\begin{tabular}{|c|c|c|}
\hline Respiratory system & Cardiovascular system & Central nervous system \\
\hline $\begin{array}{l}\text { lowering the partial pressure } \\
\text { of } \mathrm{O}_{2} \text { in arterial blood } \\
\text { (lower arterial oxygen } \\
\text { saturation - desaturation) }\end{array}$ & $\begin{array}{l}\text { cyclical variations in heart rate: } \\
\text { tachycardia, bradycardia }\end{array}$ & waking up \\
\hline $\begin{array}{l}\text { increasing the partial pressure } \\
\text { of } \mathrm{CO}_{2}\end{array}$ & $\begin{array}{l}\text { arrhythmia: additional boost } \\
\text { and supraventricular tachycardia origin } \\
\text { and the coexistence of significant hypoxemia } \\
\text { and diseases of the cardiovascular system } \\
\text { - complex arrhythmias }\end{array}$ & $\begin{array}{l}\text { excessive activation of } \\
\text { the sympathetic part } \\
\text { of autonomic nervous system }\end{array}$ \\
\hline reducing $\mathrm{pH}$ & $\begin{array}{l}\text { conduction disturbances: } \\
\text { inhibition of sinus, } \\
\text { atrioventricular block }\end{array}$ & $\begin{array}{l}\text { wide fluctuations in blood flow } \\
\text { through the central nervous system }\end{array}$ \\
\hline $\begin{array}{l}\text { periods of hyperventilation } \\
\text { after apnea }\end{array}$ & $\begin{array}{l}\text { increase in systemic arterial pressure } \\
\text { and pulmonary }\end{array}$ & breakdown of the stage of sleep \\
\hline
\end{tabular}

Possible OSA symptoms.

\section{TABLE II}

\begin{tabular}{l|l}
\hline \hline \multicolumn{1}{c|}{ At night } & \multicolumn{1}{c}{ During day } \\
\hline Loud, irregular snoring & $\begin{array}{l}\text { A feeling of lack of sleep } \\
\text { Excessive movement } \\
\text { during sleep }\end{array}$ \\
Fweating & $\begin{array}{l}\text { Drying of the mucous membranes } \\
\text { of the mouth pharynx and sore throat }\end{array}$ \\
Frequent awakenings & Head pain \\
Restless sleep & Excessive sleepiness during the day \\
Nightmares & Cognitive impairment \\
& Excessive irritability \\
& Tendency to depressive reactions
\end{tabular}

\section{Tools and methods}

Snoring is a term used to describe all sounds generated by the vibrations of partially collapsed and unstable pharyngeal airway walls and the soft plate. Snoring is a breathing noise that appears during the inspiratory and sometimes also the expiratory phase of the respiratory cycle [2]. Moreover, snoring is perceived more as a symptom or sign of development of obstructive sleep apnea syndrome in the future. Among adults who snore, two kinds of snorers can be distinguished: occasional and habitual. These two kinds of snoring could have different influence on quality rest during sleep: usually an occasional snorer has good sleep and rest but a habitual snorer is often tired after all night sleep and does not have good quality rest. Many factors influence the acoustic phenomenon of snoring. Sound of snoring might change during a single sleeping period and it may vary from night to night. Breathing by nasal, oral or both structures of upper airway, sleep stage, body position - these aspects determine quality of the sound. Snoring must be distinguished from the other sleep sounds or noises. Objective analysis of snoring is very important for treatment evaluation.

\subsection{Acoustic phenomena}

Sounds occurring during the night are produced in the vocal tract, similarly to speech. Thanks to that analogy, existing techniques for speech analysis have been applied to evaluate snoring sounds. Snoring sounds were precisely and easily detected with the use of a condenser microphone. Microphone was hung in front of the patient's mouth at a distance of about $5 \mathrm{~cm}$. The signal was recorded and sent through the analogue-digital converter directly to the computer system and subsequent analysis was performed. Acoustic analysis of sounds commenced with auditory analysis, where different types of sounds can be observed e.g. snoring, wheezing, stopped breathing etc. There are a lot of techniques and methods employed to measure the snoring. Acoustic analysis techniques give information on the mechanism, loudness, intensity and it is possible to extract relevant parameters to describe the signal [10].

Snoring and other sounds have been analyzed in the frequency and time domain and were defined with a set of quantitative sound parameters [7]. The transformation of data from the time domain to the frequency domain was carried out by the short-time Fourier transform (STFT) algorithm. Sampling frequency of the analog-to-digital converter $(44100 \mathrm{~Hz})$ determines the maximum time duration of the sample. Frequency range of $12 \mathrm{kHz}$ can completely describe the snoring phenomenon. STFT can be described using the following equation:

$$
\operatorname{STFT}_{x}^{T}=X(\tau, f)=\int_{-\infty}^{\infty} x(t) w(t-\tau) \mathrm{e}^{-\mathrm{i} 2 \pi f t} \mathrm{~d} t
$$

where $w(t)$ is the window function, commonly a Hamming window (width $N=353$ samples), centered around zero, and $x(t)$ is the signal to be transformed. $X(\tau, f)$ is essentially the Fourier transform of $x(t) w(t-\tau)$, a complex function representing the phase and magnitude of the signal over time and frequency [7, 10]. 
Visual analysis of images of acoustic signal of standard and pathological sound was comprised of observing and comparing the shape of the individual time variations, spectra and the degree of deformation. Based on time variation of the signals, two- and three-dimensional dynamic spectra and the averaged amplitude-frequency spectra, one can evaluate the signal degree of distortion. Visual analysis began with time series observations, as they represent the original image of a pathological condition. Using a computer script written in MATLAB programming environment, acoustic signal parameters were extracted and later evaluated for quantitative measurement of their similarities and differences, compared with reference signals (normal calm sleep). Different types of coordinates from the vector of characteristics are used in the comparison; therefore prior to any analysis, data normalization had to be performed. After normalization, the results were presented graphically, allowing easy identification of differences between normal breathing and snoring sounds during the night.

Figure 2 shows the difference between normal breathing and breathing with a disturbance. Performed research enabled the collection of data concerning the acoustic signal of snoring, sleep apnea etc. for a group of patients.

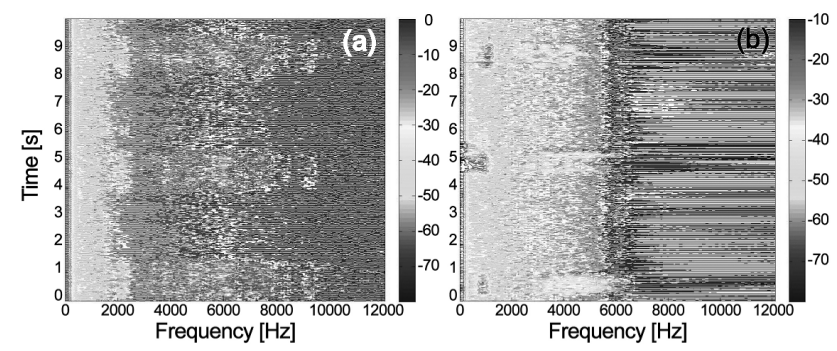

Fig. 2. STFT images show difference between normal breathing (a) and breathing with disturbance (b).

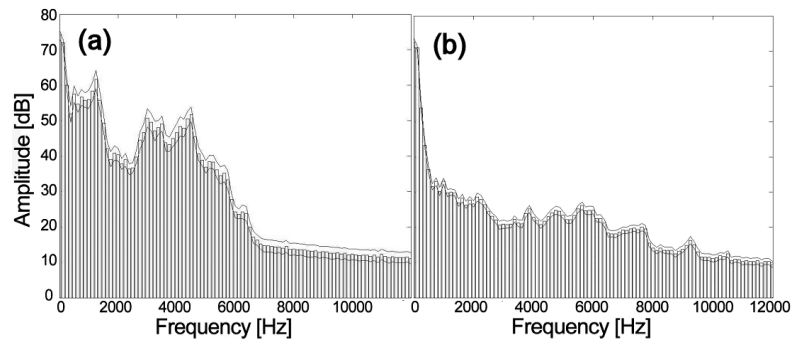

Fig. 3. (a) Events of habitual snoring. (b) Normal calm sleep, events of breathing.

Figure 3 shows a graphical representation of the power spectrum for a single breath for a snorer and a non-snorer.

Results of the acoustic signal parameters like formants of event snoring are shown below (Table III), where one can observe large differences in values e.g. the first formant.

TABLE III

Formants - acoustic parameter of snoring sound.

\begin{tabular}{c|c|c|c}
\hline \hline Formants & $\begin{array}{c}\text { Soft snorer } \\
{[\mathrm{Hz}]}\end{array}$ & $\begin{array}{c}\text { Habitual snorer } \\
{[\mathrm{Hz}]}\end{array}$ & $\begin{array}{c}\text { Normal sleep } \\
{[\mathrm{Hz}]}\end{array}$ \\
\hline F1 & 127.00 & 125.00 & 989.6 \\
F2 & 2499.00 & 1249.00 & 2058.4 \\
F3 & 2748.00 & 1874.00 & 3081.2 \\
F4 & 3123.00 & 2998.00 & 4068.4
\end{tabular}

\subsection{Electrocardiography (ECG) and $E C G$-derived respiration (EDR)}

Electrocardiogram-derived respiratory (EDR) and the pattern of heart rate variability (HRV) are obtained from the ECG recordings [6]. The ECG signal from the chest surface is disturbed by the position change of heart electrode and impedance changes associated with inhalation and exhalation. It is also possible to observe the breathing cycle: slow oscillation frequency of QRS amplitudes correspond to the breathing cycle, thus it is possible to observe these changes in ECG $[3,5]$. QRS complex is a name for the combination of three of the graphical deflections seen on a typical electrocardiogram. Amplitude method exploits the fact that the movements of the chest during respiration causes changes in the amplitude of the measured ECG signal. In inhalation phase, electrodes placed on the chest recede from the heart, thus decreasing the amplitude of the recorded signal. In exhalation chest drops, therefore the distance between the heart and the electrodes is reduced and the amplitude of the measured signal increases. Intervals method relies on getting a signal on the basis of respiratory intervals between successive heartbeats, which is represented by the QRS complex [4]. Physiological phenomenon of respiratory arrhythmia heart rate is useful in this method. During inhalation heart rate speeds up, thus spacing between successive QRS decreases. During exhalation there is opposite situation. The respiratory curve is plotted based on the inverse of spacing. EDR signal calculated within the detected QRS area, based on RS amplitude, which is measured as the difference between the minimum of the $S$ and maximum of the $R$ waves:

$$
\operatorname{amp}(i)=R_{\text {amp }}(i)-S_{\text {amp }}(i), \quad i=1,2, \ldots, n .
$$

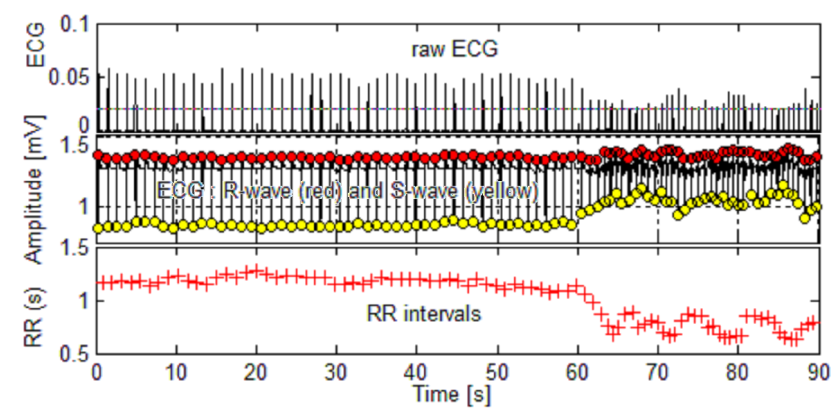

Fig. 4. RR intervals for the case of sleep apnea. 


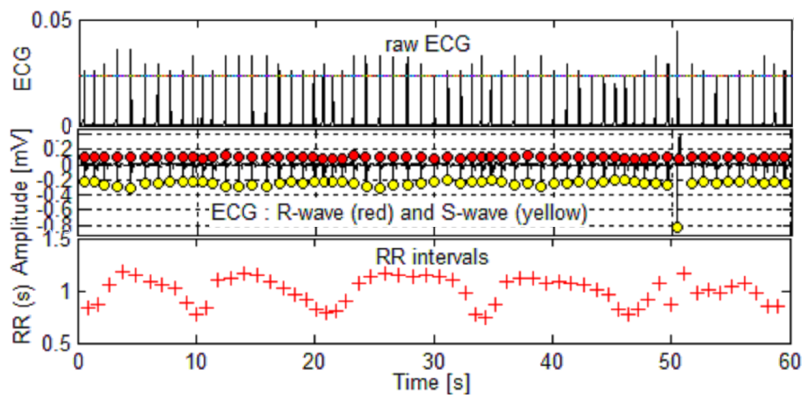

Fig. 5. RR intervals for the case of normal breath.

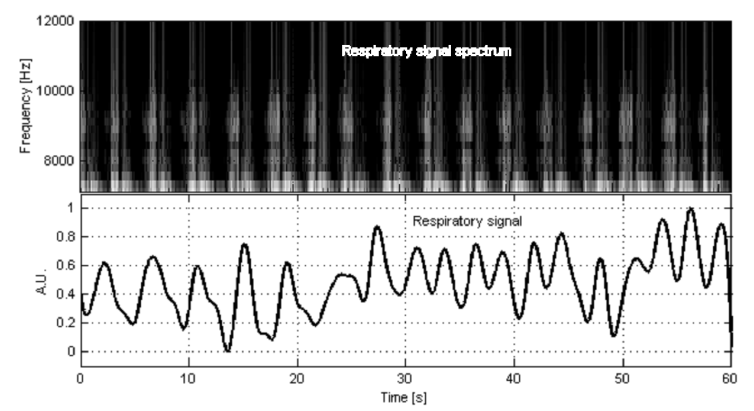

Fig. 6. Correlation of sound and ECG derived respiratory signals [9].

RR interval it is the peak of one QRS complex to the peak of the next, it is used to assess the ventricular rate. Below RR intervals for the case of sleep apnea (Fig. 4) and normal breathing (Fig. 5) are shown.

Respiratory signal analysis was performed using two described methods: acoustic analysis and EDR, and the comparison of the breath signal calculated using both methods indicates their equivalence in the assessment of breath or disturbance during sleep. Figure 6 shows the correlation of sound-derived respiratory and EDR signals from a subject breathing at $18 \mathrm{bpm}$ (beats per minute) during sleep.

From these images, the dynamic of the breathing cycle can be observed by two methods. Interpretation of recorded breathing cycle during inspiration and expiration is not easy. Lower energy in spectrogram is observed when EDR signal is decreasing. The acoustic dynamics and EDR signal can be compared for every phase (inspiration or expiration) using parameters extracted from analysis or based on images showing two methods.

\section{Conclusion}

The results of the research of acoustic and ECG-derived respiration analysis were applied as foundation for an automatic system with interpretation for cardiac activity. Two different methods for data acquisition allow the assessment of heart activity from different points of view. Thus it is possible to compare the two methods and evaluate their equivalency. Interpretation of data was based on the specific parameters extracted from automatic analysis. Close examination of parameters from snoring events and other sound signals appearing during various stages of sleep demonstrated that we can distinguish these acoustic signals into two groups: coming from a person just snoring or snoring with sleep apnea. By the simultaneous measurement of the acoustic and cardiac signals, it is possible to identify life-threatening conditions associated with respiratory arrest. Sound analysis can be used to find a correlation between disturbing sounds appearing during the night and real life-threatening situations. ECG analysis provides a complementary parameter which supports the estimation of situations dangerous to life while you sleep. However it is very important to use the described sound analysis methods as markers of abnormality in context of patient's medical history and related diagnostic tests to determine whether the patient has abnormalities or is just a healthy snorer without other disorders.

\section{Acknowledgments}

This scientific work is supported by the Polish State Committee for Scientific Research resources in years 2009-2012 as a research project No. N N518 426736.

\section{References}

[1] Y. Peng, Ch. Lin, M. Sun, C. Landis, IEEE Trans. Biomed. Circuits System 1, 217 (2007).

[2] T. Penzel, J. McNames, P. de Chazal, A. Murray, G. Moody, B. Raymond, Med. Biol. Eng. Comput. 40, 402 (2002).

[3] C. Maier, H. Dickhaus, P. Laguna, Comput. Cardiol. 32, 355 (2005).

[4] A. Kikta, P. Augustyniak, J. Med. Inform. Technol. 11, 155 (2007).

[5] S. Canisius, T. Ploch, V. Gross, A. Jerrentrup, T. Penzel, K. Kesper, in: 30th Annual Int. IEEE EMBS Conf., Canada 2008, pp. 2602, 2605.

[6] P. Langley, E.J. Bowers, A. Murray, IEEE Trans. Biomed. Eng. 57, (2010).

[7] K. Czopek, Bio-Algorithms Med-Systems 7, 37 (2011).

[8] M. Smoleń, K. Czopek, P. Augustyniak, 2, 367 (2010).

[9] M. Smoleń, K. Czopek, P. Augustyniak, Comput. Cardiol. 37, 45 (2010).

[10] T. Zielinski, Digital Signal Processing, WKŁ, Warsaw 2005 (in Polish). 